\title{
Particle velocity and acoustic impedance density of the ultrasonic field by the circular flat transducers
}

\author{
Tohru Imamura \\ National Research Laboratory of Metrology, \\ 1-1-4, Umezono, Tsukuba, Ibaraki, 305 Japan
}

(Received 23 August 1990)

\begin{abstract}
Particle velocity and acoustic impedance density of the ultrasonic field on the axis of a circular flat transducer are computed together with sound pressure. In nearfield range, interference pattern with t:ansducer distance is investigated. The phase delay of acoustic impedance density jumps always from $-\pi / 2$ to $\pi / 2$, where amplitude becomes zero. But, this regular jump is not the case for sound pressure. Next, the ultrasonic field off the axis of the circular flat transducer is investigated. It is revealed that the case on the axis is the extremity of the general ultrasonic field around the axis. The mean value over a model of receiving coaxial circular flat transducer is also computed changing the ratio of the radius $(a)$ of the circular flat transducer to the wavelength $(\lambda)$ of the ultrasonic wave. Computed amplitude of sound pressure, particle velocity and acoustic impedance density are tabulated with the normalized distance $\left(z \lambda / a^{2}\right)$. The mean amplitude of $Z$-component of particle velocity is always less than 1.0 and seems to be an appropriate response for the ultrasonic system of a pair of circular flat transducers.
\end{abstract}

Keywords: Acoustic impedance density, Particle velocity, Circular flat transducer, Ultrasonic nearfield

PACS number: 43. 20. --f

\section{INTRODUCTION}

During years of investigation for ultrasonic nearfield, only sound pressure, namely, the spatial distribution of velocity potential has long been paid attention. $^{1-6)}$ In this paper, particle velocity ${ }^{7-9)}$ and acoustic impedance density of ultrasonic field by circular flat transducers are derived and computed together with sound pressure. Sound pressure is proportional to velocity potential of ultrasonic field. Its particle velocity is space differential of velocity potential, and acoustic impedance density is quotient of sound pressure by particle velocity.

Both amplitude and phase delay are investigated changing the ratio of the radius of the circular flat transducer to the wavelength of the ultrasonic wave. The distribution on the axis of the circular flat transducer and that off the axis of it are computed, and the mean value over a model of receiving coaxial circular flat transducer is also investigated.

\section{PARTICLE VELOCITY AND ACOUSTIC IMPEDANCE DENSITY}

Medium of computation is supposed to be isotropic and non-absorptive. Coordinate system used in the computation is shown in Fig. 1. The center of the transmitter is set to be the origin. The axis of the transducers is $Z$-axis. $R$-axis is perpendicular to $Z$-axis. Distance between $\mathrm{P}$ and $\mathrm{Q}$ is called d.

The particle velocity on the transmitter being set as

$$
\dot{v}_{0} \exp (j \omega t),
$$

in which $v_{0}$ denotes amplitude, $\omega$ denotes angular velocity, the velocity potential on the receiver is expressed as 


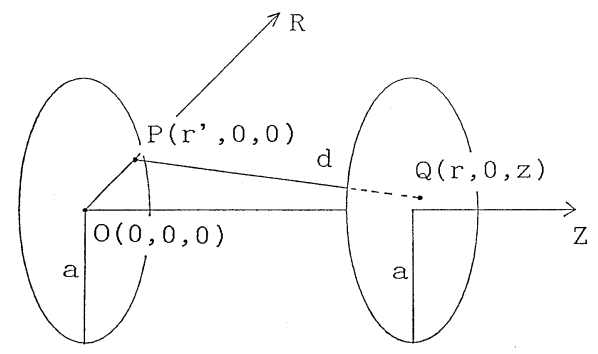

Transmitter

Receiver

Fig. 1 Coordinate system used in the computation.

$$
\begin{aligned}
\dot{\Phi} & =\iint_{S^{\prime}} \frac{\dot{v}_{0} \exp \{j(\omega t-k d)\}}{2 \pi d} d S^{\prime} \\
& =\dot{v}_{0} \exp (j \omega t) \cdot \frac{1}{2 \pi} \iint_{S^{\prime}} \frac{\exp (-j k d)}{d} d S^{\prime},
\end{aligned}
$$

in which $k$ denotes angular wave number, $S^{\prime}$ denotes transmitting area. Sound pressure can be derived as

$$
\begin{aligned}
\dot{p} & =\rho \frac{\partial \dot{\Phi}}{\partial t} \\
& =\dot{v}_{0} \exp (j \omega t) \cdot \rho c\left(\frac{j}{\lambda}\right) \iint_{S^{\prime}} \frac{\exp (-j k d)}{d} d S^{\prime} \\
& \equiv \dot{v}_{0} \exp (j \omega t) r \exp (-j \theta),
\end{aligned}
$$

in which $r$ and $\theta$ depend only on space variables.

Space differential of Eq. (2) on $Z$-axis can be represented as

$$
\begin{aligned}
\dot{v}_{z}= & -\frac{\partial \dot{\Phi}}{\partial z} \\
= & \dot{v}_{0} \exp (j \omega t) \\
& \cdot\left(\frac{1}{2 \pi}\right) \iint_{S^{\prime}} \frac{z(1+j k d) \exp (-j k d)}{d^{3}} d S^{\prime} \\
\equiv & \dot{v}_{0} \exp (j \omega t) r_{\mathrm{D}} \exp \left(-j \theta_{\mathrm{D}}\right)
\end{aligned}
$$

in which $r_{\mathrm{D}}$ and $\theta_{\mathrm{D}}$ again depend only on space variables.

Acoustic impedance density can be derived from Eqs. (3) and (4) as

$$
\begin{aligned}
\frac{\dot{p}}{\dot{v}_{z}} & =\rho c \cdot\left(\frac{2 \pi j}{\lambda}\right) \frac{\iint_{S^{\prime}} \frac{\exp (-j k d)}{d} d S^{\prime}}{\iint_{S^{\prime}} \frac{z(1+j k d) \exp (-j k d)}{d^{3}} d S^{\prime}} \\
& \equiv r / r_{\mathrm{D}} \exp \left\{-j\left(\theta-\theta_{\mathrm{D}}\right)\right\} .
\end{aligned}
$$

Ultrasonic field on the axis of the circular flat transducer is well known to be solved analytically, and Eq. (3) is expressed as

$$
\begin{aligned}
\dot{p} & =\rho \frac{\partial \dot{\Phi}}{\partial t} \\
& =\dot{v}_{0} \exp (j \omega t) \cdot \rho c\left\{\exp (-j k z)-\exp \left(-j k \sqrt{a^{2}+z^{2}}\right)\right\},
\end{aligned}
$$

and Eq. (4) can be reduced to

$$
\begin{aligned}
\dot{v}_{z}= & -\frac{\partial \dot{\Phi}}{\partial z} \\
= & \dot{v}_{0} \exp (j \omega t) \cdot\{\exp (-j k z) \\
& \left.-\frac{z}{\sqrt{a^{2}+z^{2}}} \exp \left(-j k \sqrt{a^{2}+z^{2}}\right)\right\} .
\end{aligned}
$$

From Eqs. (6) and (7), the sound pressure and the particle velocity on the axis of the circular flat transducer are computed. Acoustic impedance density is derived from dividing sound pressure by particle velocity. Ultrasonic field off the axis of the circular flat transducer is computed by a numerical method using the axial symmetry with repeated interval-halving ${ }^{9,10)}$ directly from Eqs. (3) (5).

The mean sound pressure over a model of receiving circular flat transducer is expressed as

$$
\begin{aligned}
\dot{p}_{\mathrm{m}} & =\iint_{S} \dot{p} d S \\
& =\dot{v}_{0} \exp (j \omega t) \iint_{S} r \exp (-j \theta) d S \\
& \equiv \dot{v}_{0} \exp (j \omega t) r_{\mathrm{m}} \exp \left(-j \theta_{\mathrm{m}}\right),
\end{aligned}
$$

in which $S$ denotes receiving area, and $r_{\mathrm{m}}$ and $\theta_{\mathrm{m}}$ depend only on space variables.

The mean of $Z$-component of particle velocity over a model of receiving circular flat transducer is expressed as

$$
\begin{aligned}
\dot{v}_{z \mathrm{~m}} & =\iint_{S} \dot{v}_{z} d S \\
& =\dot{v}_{0} \exp (j \omega t) \iint_{S} r_{\mathrm{D}} \exp \left(-j \theta_{\mathrm{D}}\right) d S \\
& \equiv \dot{v}_{0} \exp (j \omega t) r_{\mathrm{Dm}} \exp \left(-j \theta_{\mathrm{Dm}}\right) d S,
\end{aligned}
$$

in which $r_{\mathrm{Dm}}$ and $\theta_{\mathrm{Dm}}$ again depend only on space variables.

The mean acoustic impedance density over a model of receiving circular flat transducer is expressed as

$$
\frac{\dot{p}_{\mathrm{m}}}{\dot{v}_{z \mathrm{~m}}}=\frac{r_{\mathrm{m}}}{r_{\mathrm{Dm}}} \exp \left\{-j\left(\theta_{\mathrm{m}}-\theta_{\mathrm{Dm}}\right)\right\} .
$$




\section{T. IMAMURA: PARTICLE VELOCITY AND ACOUSTIC IMPEDANCE DENSITY}

\section{ULTRASONIC FIELI) BY A CIRCULAR FLAT TRANSDUCER}

Sound pressure, particle velocity and acoustic impedance density on the axis of the circular flat transducer are computed from Eqs. (6) and (7). Changing the ratio of the radius (a) of the circular flat transducer to the wavelength $(\lambda)$ of the ultrasonic wave, Figs. $2 \sim 10$ are derived. Figures 2, 3 and 4 show sound pressure. Figures 5, 6 and 7 show particle velocity. And Figs. 8, 9 and 10 show acoustic impedance density. With increasing $a / \lambda$, ultrasonic field has more complexity. Figures 2, 5 and 8 are the case when $a / \lambda=1.71$. In the origin, the amplitude of the particle velocity is 1.0 , and the
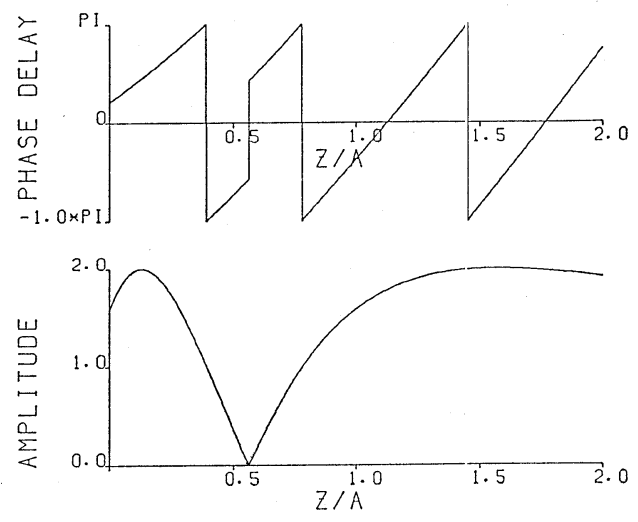

Fig. 2 Sound pressure on the axis of the circular flat transducer $(a / \lambda=1.71)$.

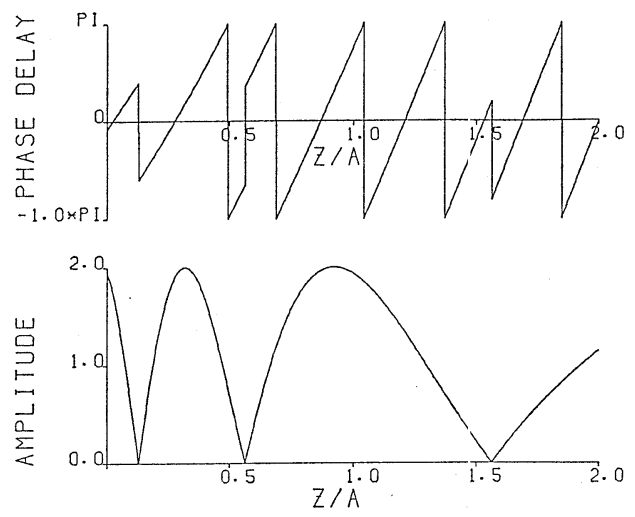

Fig. 3 Sound pressure on the axis of the circular flat transducer $(a / \lambda=3.42)$.

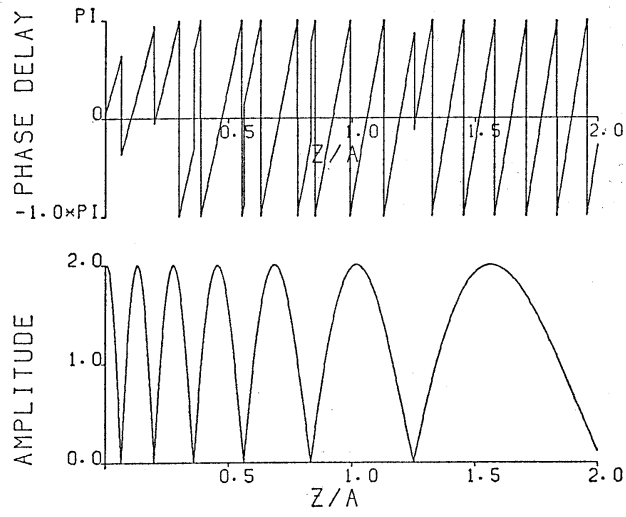

Fig. 4 Sound pressure on the axis of the circular flat transducer $(a / \lambda=8.55)$.
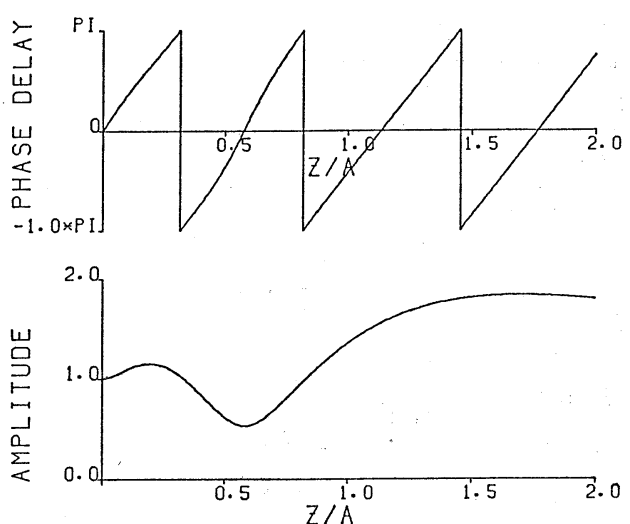

Fig. 5 Particle velocity on the axis of the circular flat transducer $(a / \lambda=1.71)$.
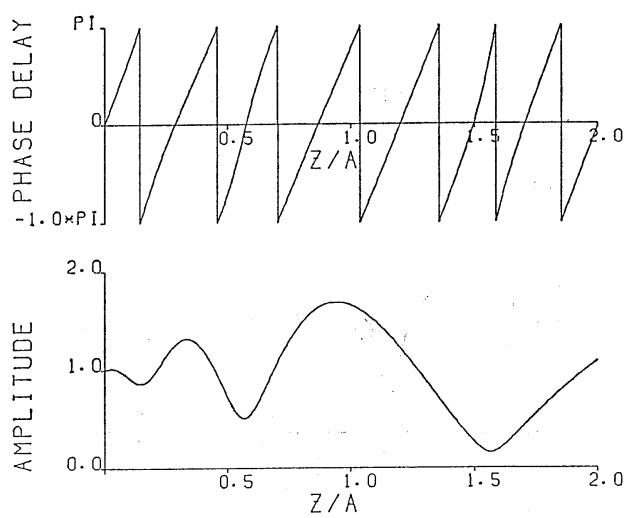

Fig. 6 Particle velocity on the axis of the circular flat transducer $(a / \lambda=3.42)$. 


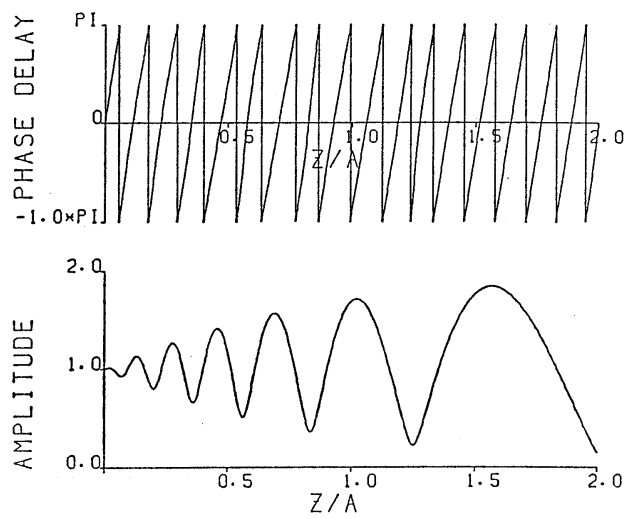

Fig. 7 Particle velocity on the axis of the circular flat transducer $(a / \lambda=8.55)$.
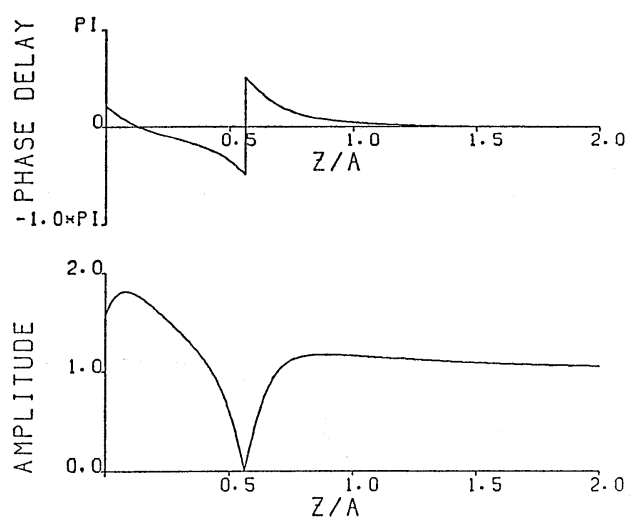

Fig. 8 Acoustic impedance density on the axis of the circular flat transducer $(a / \lambda$ $=1.71$ ).
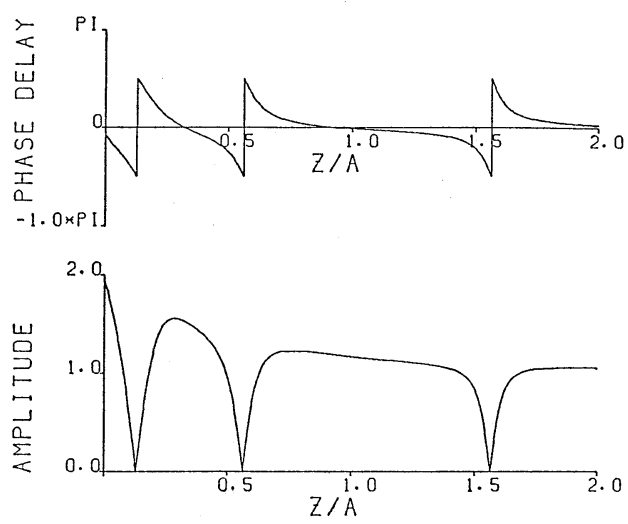

Fig. 9 Acoustic impedance density on the axis of the circular flat transducer $(a / \lambda$ $=3.42$ ).
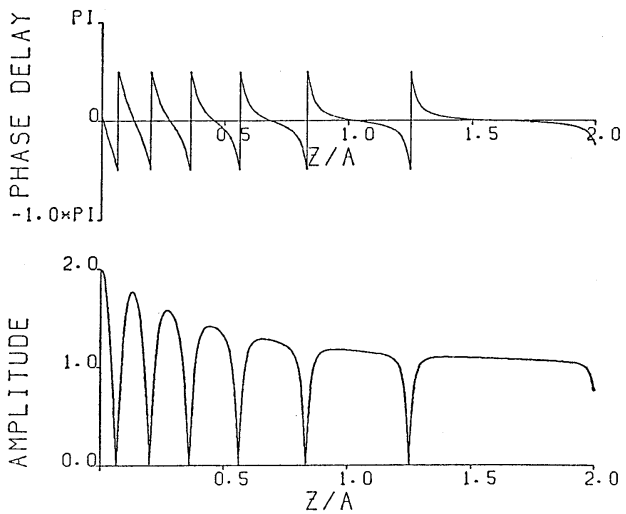

Fig. 10 Acoustic impedance density on the axis of the circular flat transducer $(a / \lambda$ $=8.55$ ).

phase delay of it is 0.0 . The amplitude of the sound pressure reaches zero when $z / a$ is about 0.6. The phase delay of it jumps at this point. The jump of the acoustic impedance density is from $-\pi / 2$ to $\pi / 2$. Figures 3,6 and 9 are the case when $a / \lambda=3.42$. They have three jump points. The jumps of the sound pressure are irregular. On the other hand, those of the acoustic impedance density are always from $-\pi / 2$ to $\pi / 2$. Figures 4,7 and 10 are the case when $a / \lambda=8.55$, and have six jump points showing a similar tendency as before. From amplitude distribution of particle velocity, how interference occurs with distance from transducer can be found. With increasing $a / \lambda$, phase delay of acoustic impedance density has more number of constant jumps from $-\pi / 2$ to $\pi / 2$.

In the next, we observe how sound pressure, particle velocity and acoustic impedance density behave off the axis of the circular flat transducer. Figures 11, 12 and 13 show sound pressure. Figures 14, 15 and 16 show particle velocity. And Figs. 17, 18 and 19 show acoustic impedance density. All of these figures are in the case where $a / \lambda=1.71$. Figures 11,14 and 17 are the case when the parallel distance $(r)$ from the axis of the circular flat transducer is $0.05 a$. The amplitude of the sound pressure does not reach zero, but it has a deep valley when $z / a$ is about 0.6. At this point, the phase delay of the sound pressure has a very sharp increase. The phase delay of the acoustic impedance density does not jump, but has a remarkable increase from minus to plus. The particle velocity does not have 

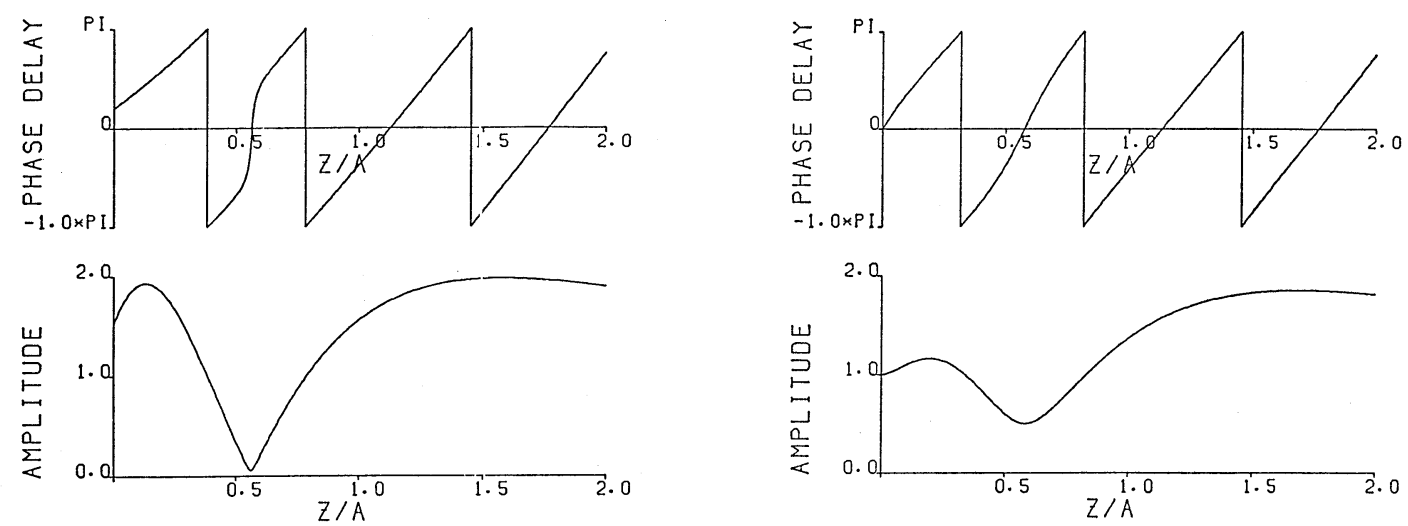

Fig. 11 Sound pressure off the axis of the circular flat transducer $(a / \lambda=1.71$, $r=0.05 a)$.

Fig. 14 Particle velocity off the axis of the circular flat transducer $(a / \lambda=1.71$, $r=0.05 a$ ).
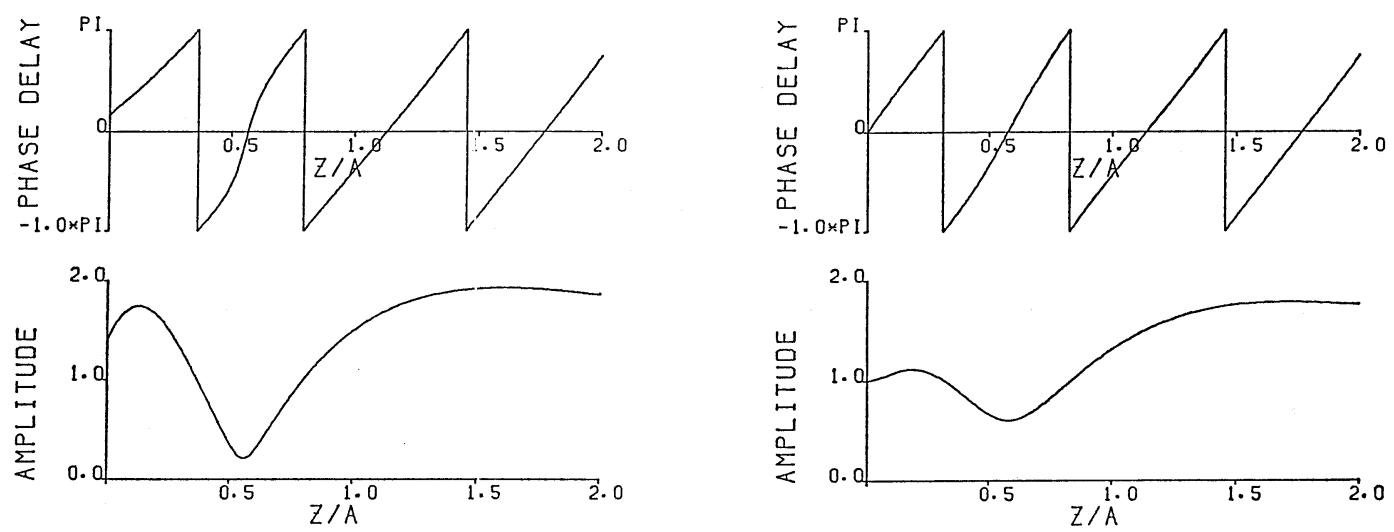

Fig. 12 Sound pressure off the axis of the circular flat transducer $(a / \lambda=1.71$, $r=0.1 a)$.

Fig. 15 Particle velocity off the axis of the circular flat transducer $(a / \lambda=1.71$, $r=0.1 a)$.
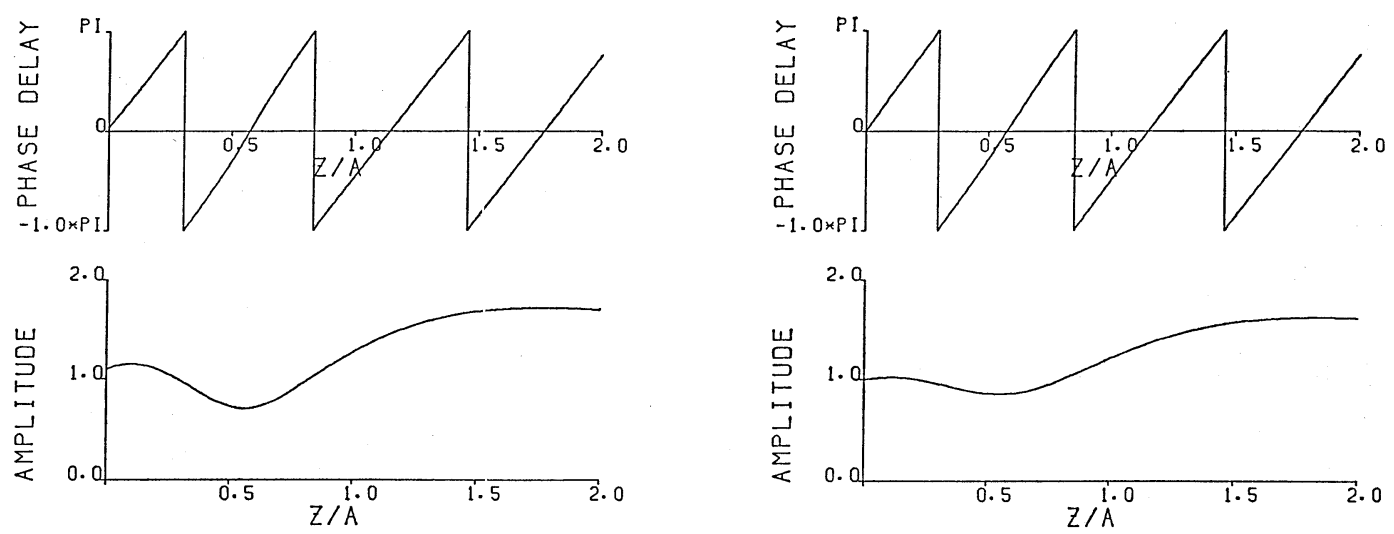

Fig. 13 Sound pressure off the axis of the circular flat transducer $(a / \lambda=1.71$, $r=0.2 a)$.

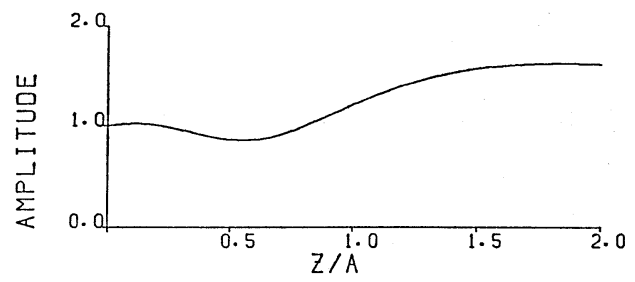

Fig. 16 Particle velocity off the axis of the circular flat transducer $(a / \lambda=1.71$, $r=0.2 a)$. 


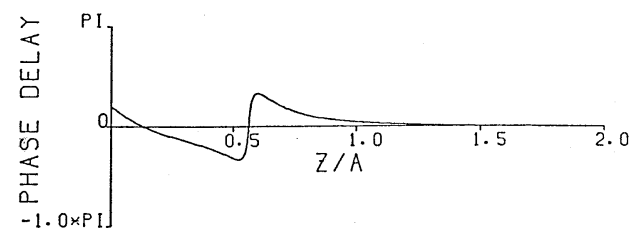

much difference from that on the axis of the circular flat transducer. Figures 12, 15 and 18 are the case when $r=0.1 a$. The feature becomes more mild, but it keeps similar tendency as before. Figures 13, 16 and 19 are the case when $r=0.2 a$, and the feature becomes weak. In general, the ultrasonic field on the axis of the circular flat transducer is the extremity of the general ultrasonic field around the axis of it.

As a whole, on the axis of the circular flat transducer, the phase delay of the sound pressure shows a peculiar feature, but it can be explained appropriately from the particle velocity and the acoustic impedance density.

Fig. 17 Acoustic impedance density off the axis of the circular flat transducer $(a / \lambda$ $=1.71, r=0.05 a$ ).

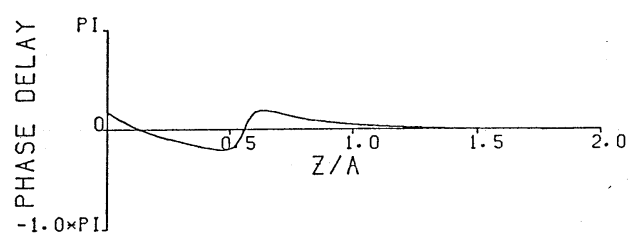

\section{MEAN VALUE OVER A MODEL OF RECEIVING COAXIAL CIRCULAR FLAT TRANSDUCER}

Mean values over a model of receiving coaxial circular flat transducer are also computed by the numerical method from Eqs. (8) (10). Table 1 shows the mean amplitude of sound pressure over a model of receiving coaxial circular flat transducers.

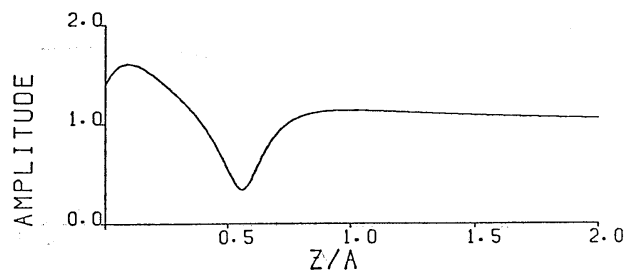

Fig. 18 Acoustic impedance density off the axis of the circular flat transducer $(a / \lambda$ $=1.71, r=0.1 a$ ).

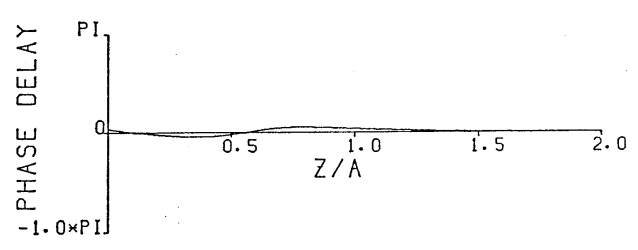
In the table, variation by change of $a / \lambda$ with same normalized distance $\left(z \lambda / a^{2}\right)$ is shown. When normalized distance equals 1.0 , the amplitude varies from 0.806 to 0.959 according to the difference of the value of $a / \lambda$ from 0.342 to 17.1. In all the cases when normalized distance is from 0.02 to 10.0 , the amplitude varies according to the difference of the value of $a / \lambda$, even if the normalized distance is the same. In general, the amplitude gets small having a few exceptions with the increase of the normalized distance. Table 2 presents the mean amplitude of $Z$-component of particle velocity over a model of receiving coaxial circular flat transducers. As is the sound pressure, the particle velocity has variation according to the value of $a / \lambda$ even if the normalized distance is the same. This has the similar tendency as the sound pressure. But, all data of the amplitude of the particle velocity have the value less than 1.0. It seems that the mean particle velocity is an appropriate response for the ultrasonic system of a pair of circular flat transducers. Table 3 shows the mean amplitude of acoustic impedance density over a model of receiving coaxial circular flat transducers. The value reaches 1.0 when both distance between transducers and $a / \lambda$ are large. The phase delay in this range is revealed to be 0.0. This is equal to that the feature of the sound pressure and

Fig. 19 Acoustic impedance density off the axis of the circular flat transducer $(a / \lambda$ $=1.71, r=0.2 a$ ). 


\section{T. IMAMURA: PARTICLE VELOCITY AND ACOUSTIC IMPEDANCE DENSITY}

Table 1 Amplitude of the mean sound pressure over a model of receiving coaxial circular flat transducers.

\begin{tabular}{rrrrrrr}
\hline \multirow{2}{*}{$z \lambda / a^{2}$} & \multicolumn{7}{c}{$a / \lambda$} \\
\cline { 2 - 7 } & 0.342 & 0.855 & 1.71 & 3.42 & 8.55 & 17.1 \\
\hline 0.02 & 1.173 & 1.023 & 0.968 & 0.975 & 0.971 & 0.970 \\
0.05 & 1.164 & 1.005 & 0.952 & 0.951 & 0.953 & 0.951 \\
0.1 & 1.151 & 0.978 & 0.941 & 0.940 & 0.931 & 0.932 \\
0.2 & 1.125 & 0.931 & 0.931 & 0.913 & 0.907 & 0.903 \\
0.5 & 1.056 & 0.836 & 0.846 & 0.853 & 0.859 & 0.858 \\
1.0 & 0.959 & 0.807 & 0.806 & 0.818 & 0.810 & 0.809 \\
2.0 & 0.801 & 0.818 & 0.774 & 0.758 & 0.753 & 0.752 \\
5.0 & 0.505 & 0.543 & 0.549 & 0.551 & 0.551 & 0.551 \\
10.0 & 0.293 & 0.302 & 0.304 & 0.304 & 0.304 & 0.304 \\
\hline
\end{tabular}

Table 2 Amplitude of the mean particle velocity over a model of receiving coaxial circular flat transducers.

\begin{tabular}{ccccccc}
\hline \multirow{2}{*}{$z \lambda / a^{2}$} & \multicolumn{7}{c}{$a / \lambda$} \\
\cline { 2 - 7 } & 0.342 & 0.855 & 1.71 & 3.42 & 8.55 & 17.1 \\
\hline 0.02 & 0.981 & 0.972 & 0.967 & 0.967 & 0.968 & 0.969 \\
0.05 & 0.962 & 0.950 & 0.947 & 0.949 & 0.951 & 0.951 \\
0.1 & 0.939 & 0.927 & 0.928 & 0.931 & 0.931 & 0.932 \\
0.2 & 0.904 & 0.896 & 0.904 & 0.906 & 0.906 & 0.903 \\
0.5 & 0.838 & 0.836 & 0.846 & 0.852 & 0.858 & 0.858 \\
1.0 & 0.769 & 0.791 & 0.798 & 0.814 & 0.810 & 0.809 \\
2.0 & 0.673 & 0.771 & 0.764 & 0.756 & 0.752 & 0.752 \\
5.0 & 0.467 & 0.531 & 0.546 & 0.550 & 0.551 & 0.551 \\
10.0 & 0.285 & 0.300 & 0.303 & 0.304 & 0.304 & 0.304 \\
\hline
\end{tabular}

Table 3 Amplitude of the mean acoustic impedance density over a model of receiving coaxial circular flat transducers.

\begin{tabular}{rrrrrrr}
\hline \multirow{2}{*}{$z \lambda / a^{2}$} & \multicolumn{7}{c}{$a / \lambda$} \\
\cline { 2 - 7 } & 0.342 & 0.855 & 1.71 & 3.42 & 8.55 & 17.1 \\
\hline 0.02 & 1.195 & 1.053 & 1.001 & 1.008 & 1.002 & 1.001 \\
0.05 & 1.210 & 1.058 & 1.005 & 1.002 & 1.002 & 1.000 \\
0.1 & 1.226 & 1.055 & 1.015 & 1.010 & 1.000 & 1.000 \\
0.2 & 1.245 & 1.039 & 1.030 & 1.009 & 1.001 & 1.000 \\
0.5 & 1.260 & 0.999 & 1.000 & 1.002 & 1.001 & 1.000 \\
1.0 & 1.246 & 1.020 & 1.010 & 1.005 & 1.001 & 1.000 \\
2.0 & 1.191 & 1.061 & 1.013 & 1.003 & 1.000 & 1.000 \\
5.0 & 1.083 & 1.022 & 1.006 & 1.002 & 1.000 & 1.000 \\
10.0 & 1.029 & 1.006 & 1.002 & 1.000 & 1.000 & 1.000 \\
\hline
\end{tabular}


that of the particle velocity does not have difference. Namely, the ultrasonic field can be treated as farfield or as plane wave.

\section{CONCLUSION}

Particle velocity and acoustic impedance density of circular flat transducers are computed together with sound pressure. With increasing $a / \lambda$, ultrasonic field has more complexity. From the amplitude distribution of the particle velocity on the axis of the circular flat transducer, how interference occurs with distance from transducer can be found. Phase delay of acoustic impedance density on the axis of the circular flat transducer has constant jumps from $-\pi / 2$ to $\pi / 2$, which is not true for sound pressure. This is the extremity of the general ultrasonic field around the axis of the circular flat transducer. As a whole, on the axis of the circular flat transducer, the phase delay of the sound pressure shows a peculiar feature, but it can be explained appropriately from the particle velocity and the acoustic impedance density. The mean value over a model of receiving coaxial circular flat transducer is also computed by a numerical method, and the amplitude of the sound pressure, that of the particle velocity and that of the acoustic impedance density are tabulated by the normalized distance $\left(z \lambda / a^{2}\right)$ with change of the value of $a / \lambda$. When normalized distance is from 0.02 to 10.0 , the mean amplitude of sound pressure and those of particle velocity vary according to the difference of the value of $a / \lambda$, even if the normalized distance is the same. The mean amplitude of $Z$-component of particle velocity is always less than 1.0 and seems to be an appropriate response for the ultrasonic system of a pair of circular flat transducers.

\section{REFERENCES}

1) A. O. Williams, Jr., "The piston source at high frequencies," J. Acoust. Soc. Am. 23, 1-6 (1951).

2) H. Seki, A. Granato, and R. Truell, "Diffraction effects in the ultrasonic field of a piston source and their importance in the accurate measurement of attenuation," J. Acoust. Soc. Am. 28, 230-238 (1956).

3) K. Yamada and Y. Fujii, "Acoustic response of a circular receiver to a circular source of different radius," J. Acoust. Soc. Am. 40, 1193-1194 (1966).

4) J. Zemanek, "Beam behavior within the nearfield of a vibrating piston," J. Acoust. Soc. Am. 49, 181-191 (1971).

5) A. S. Khimunin, "Ultrasonic propagation parameter measurements incorporating exact diffraction corrections," Acustica 39, 87-95 (1978).

6) T. Hasegawa, K. Matsuzawa, N. Inoue, and A. Yanagihara, "Ultrasonic scattering by a rigid sphere in the nearfield of a circular piston," J. Acoust. Soc. Jpn. (E) 6, 9-14 (1985).

7) T. Imamura and K. Iizuka, "Effect of beam divergence on ultrasonic attenuation measurement," Proc. 21st Jpn. Natl. Congr. Appl. Mech. (Univ. of Tokyo Press, Tokyo, 1973), pp. 167-176.

8) T. Imamura, "Numerical analysis of ultrasonic transmission system of rectangular transducers; Effect of translation, rotation and tilt," Bull. NRLM 33, 205-211 (1984).

9) T. Imamura, "Precise computation for the response of ultrasonic transmission systems by numerical multiple integration," J. Acoust. Soc. Jpn. (E) 7, 261-257 (1986).

10) B. Carnahan, H. A. Luther, and J. O. Wilkes, Applied Numerical Methods (John Wiley \& Sons, New York, 1969), p. 90.

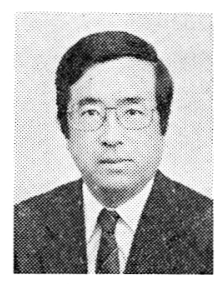

Tohru Imamura was born in $\mathrm{Na}$ goya, Japan on September 29, 1945. He received the B. Sci. from the University of Tokyo in 1969. Since then, he has been with the National Research Laboratory of Metrology, AIST, MITI. His interests are in numerical analysis of ultrasonic propagation, ultrasonic measurement of materials, and history and role of standards. 